\title{
Trauma Patient Unplanned Hospital Re-Admissions
}

\author{
Thomas J. Esposito*, Evelyn Clark-Kula, Melissa Crowe, Linda Galambos, \\ Loubna Salameh, Janice Gillespie \\ Loyola University Medical Center, Maywood, USA \\ Email: *tesposi@lumc.edu
}

Received June 3, 2012; revised July 5, 2012; accepted July 15, 2012

\begin{abstract}
Introduction: Performance monitoring and performance improvement (PI) are increasingly important. Little is known regarding unplanned re-admission (UPR) in trauma patients. This study characterizes UPRs at one institution. Methods: Retrospective descriptive review of UPR to a Level I Trauma Center Information was obtained on: initial trauma diagnoses, diagnosis precipitating UPR, discharge interval, treatment rendered and length of stay (LOS) during both encounters, and PI committee judgments. Characteristics of UPR patients were determined and compared to those of all discharged patients. Descriptive statistics were applied. Results: Over 2.5 years there were 2827 discharges and 58 UPR (2\%). The majority of original diagnoses were related to blunt trauma and head injuries. UPR occurred at a median of 3 days, with 54\% re-admitted to the trauma service. Operative rate for UPR patients during the initial admission was $48 \%$ with $28 \%$ requiring operation on the UPR. Headache and wound issues were responsible for $42 \%$ of UPR. Diagnosis precipitating UPR was primarily related to post-operative complications in $26 \%$ of all UPR and $57 \%$ of those undergoing operation on the initial admission. Median LOS for UPR was 3 days with ICU care being required by $13 \%$. Of all UPRs, 33\% were attributable to opportunities for improved care (OFI) during the first admission. Identified OFIs were related to errors in technique (53\%), errors in judgment (27\%), and system issues (20\%). Of UPR without OFI, 87\% were related to disease and 13\% systems issues. Conclusion: UPR at a Level I trauma center is rare, occurs shortly after discharge, is brief in duration and usually related to postoperative wound issues or headache. Post operative patients seem at greater risk for UPR. While most UPR are considered non-preventable, attention to discharge instructions, patient education, resident education and supervision and outpatient support, may obviate a number of preventable UPRs.
\end{abstract}

Keywords: Trauma Patients; Unplanned Hospital Readmissions; Preventable Complications; Performance Improvement

\section{Introduction}

Performance monitoring for benchmarking and performance improvement (PI) purposes are increasingly important in the era of healthcare reform and "pay for performance". Readmission to the hospital after an initial encounter is fast becoming an important quality indicator for surgical patients [1]. While information on the nature and volume of acute trauma inpatient complications is becoming more prevalent [2-4], less is known regarding the rate and causes of unplanned re-admission (UPR) to the hospital after discharge in trauma patients. Marcin and co-authors noted a $4.7 \%$ re-admission rate in the 16 65 age groups and a 9\% rate of re-admission in elderly patients greater than age 65 in their study of trauma patient volume as it related to mortality and re-admission rates [5]. Cardenas, et al. conducted a multi-center analysis of the etiology and incidence of re-hospitalization after

*Corresponding author. traumatic spinal cord injury and found the leading cause of re-hospitalization was related to diseases of the genitourinary system [6]. Battistella and colleagues have produced the most extensive analysis of outpatient complications resulting in hospital re-admission after trauma [7]. In that retrospective study, $1.4 \%$ of discharged patients required re-admission with diagnoses related to wounds, abdominal complaints, pulmonary issues, and thromboembolic complications. In that series, $24 \%$ of re-admitted patients required an operation. Risk factors for re-admission included: operation during the first hospitalization; penetrating injury; and advanced age. In 1984, Anderson estimated that Medicare would spend nearly 8 billion dollars or $24 \%$ of total inpatient expenditures on re-admissions within 60 days of discharge [8]. He further postulated that even a small decrease in re-admissions could result in substantial savings to the Medicare system. These conclusions can easily be extrapolated to the general health care system and trauma system. The purpose of this study 
is to provide further information and characterization of unplanned re-admissions after hospitalization for trauma in an effort to identify strategies to avert this additional drain on trauma center resources.

\section{Methods}

As part of a performance improvement project, a retrospective descriptive review of unplanned re-admission to the hospital at a level I trauma center after primary admission for trauma care over a 2.5 year period was carried out. Patients were included only if the UPR occurred within 30 days of discharge. Medical records of all consecutive trauma patient discharges were abstracted to obtain information on: demographics, payor mix, initial trauma diagnosis; diagnoses precipitating re-admission; interval between first discharge and secondary admission; treatment rendered, including operative interventions during both encounters; primary and secondary admission length of stay (LOS), ICU admission and LOS on primary and re-admission and PI Committee judgment on preventability/opportunity for improvement (OFI) related to the re-admission.

The Trauma Program Performance Improvement Workgroup is a multidisciplinary committee which is composed of trauma surgeons, nurse practitioners, a clinical pharmacist, trauma program manager, trauma registry coordinator and performance improvement coordinator, among others. New admissions to the trauma service, both acute and re-admissions as well as re-admissions to other services when known, are reviewed for standard complications reported to the $\mathrm{NTDB}^{\circledR}$ as well as other pertinent complications and systems issues. Preventability judgments and opportunities for improvement are determined by consensus majority opinion. OFI does not necessarily equate with preventability. Results of workgroup proceedings are entered into the trauma registry. For the purposes of this study, the aforementioned information was gleaned from the trauma registry and descriptive statistics were applied to the data.

Finally, an operative index, i.e. number of operations per patient operated upon during the first admission, was calculated. This measure was calculated using two methods. The first utilized only those patients who underwent one or more operations during the hospitalization being analyzed (initial vs UPR) as a denominator. The second utilized the entire number of patients in each group being analyzed for a denominator.

\section{Results}

Atotal of 2827 patients were discharged over the 30-month study period. During that same period, 58 patients were re-admitted (2\%) accounting for 61 separate UPR episodes. Multiple re-admissions in the same patient were counted separately and three patients had two re-admissions each. The primary reason for original admission was related to blunt trauma in 43 of the UPRs (70\%) and penetrating trauma in 15 patients (30\%) while for the entire group of discharges, $85 \%$ sustained blunt injury and $15 \%$ penetrating injuries. The majority of primary diagnoses were related to head injuries. Twenty-six percent of all discharged patients sustained a head injury while 28\% of patients with UPRs had initially sustained head injuries.

Mean age for all discharged patients was 33.5 and for the UPR patient group it was 34.8. The proportion of patients aged 65 or older was the same in both groups, that being $12 \%$. Of all discharged patients, $70 \%$ were male whereas for UPR patients $86 \%$ were male. Mean ISS for all discharged patients was 8 and for UPRs mean ISS from initial admission was 10 . Mean LOS for primary admission in all discharges was 4.5 days (median, two days). In the UPR patient group, mean hospital LOS on first admission was 8 days (median, four days). Considering all discharged patients, $40 \%$ had an ICU stay on the first encounter. In the UPR group, $57 \%$ of patients had ICU care on the primary admission. The median interval between discharge and UPR was 3 days (mean 6; range $<1$ - 28). Ninety-seven percent of the 61 UPRs occurred within 14 days of discharge and all occurred within 28 days. Summary of these comparative data are shown in Table 1.

Table 1. Comparative demographic and clinical data on all discharges and unplanned readmissions (UPR).

\begin{tabular}{|c|c|c|}
\hline & $\begin{array}{c}\text { All } \\
\text { Discharges }\end{array}$ & UPRs \\
\hline Cases & 2827 & $61(2 \%)$ \\
\hline Male & $70 \%$ & $86 \%$ \\
\hline Mean Age (Median) & $35.5(29)$ & $34.8(27.5)$ \\
\hline 65 or Greater & $12 \%$ & $12 \%$ \\
\hline Mean ISS & 8 & 10 \\
\hline Penetrating Injury & $15 \%$ & $26 \%$ \\
\hline Head Injury & $26 \%$ & $28 \%$ \\
\hline $\begin{array}{l}\text { Hospital LOS (Mean/Median Days) on } \\
\text { Primary Admission }\end{array}$ & $4.5 / 2$ & $8 / 4$ \\
\hline ICU Stay on Primary Admission & $40 \%$ & $57 \%$ \\
\hline $\begin{array}{l}\text { ICU LOS on Primary Admission } \\
\text { (Mean/Median Days) }\end{array}$ & $4.7 / 2$ & $4.3 / 3$ \\
\hline Hospital LOS on UPR (Mean/Median Days) & - & $6 / 3$ \\
\hline $\begin{array}{l}\text { *Interval between Primary Admission \& } \\
\text { UPR (Median Days) }\end{array}$ & - & 3 \\
\hline ICU Stay on UPR & - & $13 \%$ \\
\hline ICU LOS on UPR (Mean/Median Days) & - & 14 \\
\hline
\end{tabular}

\footnotetext{
${ }^{*} 97 \%$ within 14 days.
} 
Payor mix comparison between the two groups is shown in Table 2. Self-pay patients were over represented in the UPR group compared to all discharges. Discharge disposition after the first admission for the two groups is noted in Table 3. The majority in both groups were discharged home after their initial hospital stay, $77 \%$ of all discharged patients and $81 \%$ of the 58 UPR patients.

Operative intervention was performed on 28 of the 58 UPR patients (48\%) during the first admission the majority being orthopedic, abdominal or chest procedures. Operative rate for all initial trauma contacts was 36\% (1022/2827 patients). The operative rate in the re-admitted patients during the UPR was $28 \%$ with 1 patient requiring 2 operations during the re-admission period. These operations on the re-admissions were for diagnoses either related to or unrelated to causes of the first admission and were more often neurosurgical and abdominal rather than orthopedic. Operative index results are presented in Tables 4 and 5.

Table 2. Payor mix comparison between all discharges and UPR group.

\begin{tabular}{ccc}
\hline & All Discharges & UPR \\
\hline Commercial & $35 \%$ & $21 \%$ \\
Medicaid & $19 \%$ & $19 \%$ \\
Medicare & $12 \%$ & $10 \%$ \\
Self Pay & $30 \%$ & $47 \%$ \\
Workman’s Comp & $4 \%$ & $3 \%$ \\
Other & $1 \%$ & - \\
\hline
\end{tabular}

Table 3. Discharge disposition after initial hospital stay.

\begin{tabular}{ccc}
\hline & All Discharges & UPRs \\
\hline Home & $77 \%$ & $81 \%$ \\
Rehabilitation Center & $13 \%$ & $16 \%$ \\
SNF & $3 \%$ & $3 \%$ \\
Psychiatric Hospital & $1 \%$ & - \\
Jail/Prison & $0.3 \%$ & - \\
AMA & $1 \%$ & - \\
Morgue & $4 \%$ & - \\
\hline
\end{tabular}

Table 4. Comparison of operative data for all discharges and UPR group.

\begin{tabular}{ccc}
\hline & All Discharges & UPRs \\
\hline Operative Rate Primary Admission & $36 \%$ & $48 \%$ \\
No Operation & $1805(64 \%)$ & $30(52 \%)$ \\
One Operation & $708(25 \%)$ & $23(40 \%)$ \\
More than One Operation & $1652(11 \%)$ & $5(8 \%)$ \\
Operative Index & $1.6(0.6)$ & $1.4(0.7)$ \\
Operative Rate During UPR & - & $28 \%$ \\
\hline
\end{tabular}

The diagnoses or complaints precipitating re-admission are presented in Table 6. The majority of the 61 re-admissions (42\%) were related to wound issues and headache. Post-operative complications from an operation on the first trauma admission were the cause of re-admission in $26 \%$ of all 58 UPR patients and 57\% of those 28 UPR patients undergoing operation on the first trauma admission. Median LOS for UPR was 3 days (range 1 - 83). ICU care was required by $13 \%$ of re-admissions with a median ICU stay of 4 days (range 1 - 65).

Table 5. Distribution of operation type during primary admission and for the UPR encounter.

\begin{tabular}{ccc}
\hline & Primary Admission \\
\hline & All Discharges & UPR Group \\
\hline Orthopedics & $39 \%$ & $23 \%$ \\
Abdomen & $17 \%$ & $37 \%$ \\
Chest & $6 \%$ & $11 \%$ \\
Neurosurgery & $7 \%$ & $3 \%$ \\
Spine & $2 \%$ & $1 \%$ \\
CV/PV & $6 \%$ & $9 \%$ \\
Facial & $4 \%$ & $1 \%$ \\
Other & $19 \%$ & $15 \%$ \\
\hline & UPR Hospitalization & \\
\hline Orthopedics & - & $8 \%$ \\
Abdomen & - & $19 \%$ \\
Chest & - & $8 \%$ \\
Neurosurgery & - & $14 \%$ \\
Spine & - & $0 \%$ \\
CV/PV & - & $31 \%$ \\
Facial & - & $0 \%$ \\
Other & - & $19 \%$ \\
\hline
\end{tabular}

Table 6. Presenting complaint or diagnosis of patients with UPR.

\begin{tabular}{cc}
\hline Presenting Complaint or Diagnosis & $\mathrm{n}(\%)$ \\
\hline Wound Issue & $16(26 \%)$ \\
Headache & $10(16 \%)$ \\
Missed Injury & $6(10 \%)$ \\
Pain & $5(8 \%)$ \\
Bleeding & $4(7 \%)$ \\
Dyspnea & $3(5 \%)$ \\
CHF & $2(3 \%)$ \\
Ileus & $2(3 \%)$ \\
Hyponatremia & $2(3 \%)$ \\
Fever & $2(3 \%)$ \\
Social Issues & $2(3 \%)$ \\
Mental Status Changes & $2(3 \%)$ \\
Dehydration & $1(2 \%)$ \\
DVT & $1(2 \%)$ \\
Syncope & $1(2 \%)$ \\
Foot Drop & $1(2 \%)$ \\
\hline
\end{tabular}


Performance improvement review of all re-admitted cases revealed that $33 \%$ were judged preventable or associated with an opportunity for improvement in care during the first admission. There were more OFIs noted in patients with UPR after surgery on initial admission (43\%) than in those without surgery on that index admission (18\%). The majority of OFIs in cases of clinical complications were related to error in technique (53\%). Errors in technique predominated in post-surgical UPRs. Errors in judgment were identified in $27 \%$ of the UPR cases and systems issues were implicated as the reason for the UPR in $20 \%$ of cases. Most judgment errors and systems issues occurred in UPRs without surgery on the index admission. The majority of the UPRs without OFI were attributed to the nature of disease (87\%) with $13 \%$ attributed to systems issues which were beyond the scope of the trauma program's control (Tables 7 and 8 ).

Missed injuries were present in ten patients (16\%), nine of which were in UPRs that were not post-op. Three (30\%) were judged to represent an OFI (Table 9). There were no deaths in the UPR group.

Table 7. Analysis of UPR performance improvement findings.

\begin{tabular}{ccc}
\hline & Post-Op & Non-Op \\
\hline UPR Readmission Class & $46 \%$ & $54 \%$ \\
OFI & $43 \%$ & $18 \%$ \\
& Overall OFI rate for UPR—33\% & \\
\hline
\end{tabular}

Table 8. Analysis of preventability and opportunities for improvement (OFI).

\begin{tabular}{cc}
\hline \multicolumn{2}{c}{ Preventable/OFI Cases } \\
\hline Error in Technique & $53 \%$ \\
Error in Judgement & $27 \%$ \\
Systems Issue & $20 \%$ \\
Non-Preventable/No OFI Cases \\
\hline Nature of Disease & $87 \%$ \\
System Issues beyond control & $13 \%$ \\
\hline
\end{tabular}

Table 9. Missed injuries found on UPR and preventability/OFI findings.

\begin{tabular}{cc}
\hline Injury & Preventable/OFI \\
\hline Urethral Injury & Yes \\
SDH/Skull Fx & Yes \\
Ulnar Diaphysis Fx & No \\
Rib Fx & No \\
ICH & No \\
Clavicle Fx/Hyponatremia & Yes \\
Clavicle Fx & No \\
Puncture Wound & No \\
Skull Fx & No \\
DVT & No \\
\hline
\end{tabular}

The majority of cases (54\%) were re-admitted to the trauma service. Twenty percent were re-admitted to nonsurgical services, predominantly pediatrics (Table 10).

\section{Discussion}

This review shows that unplanned re-admission after initial treatment at a Level I Trauma Center and teaching hospital is relatively rare and predominantly not preventable. In this study, these secondary encounters occurred shortly after the first discharge, were brief in duration, and usually related to surgical wound issues or headache.

Patients with penetrating trauma appeared to be over presented in the UPR group in comparison to their representation in the group of all discharged patients (26\% vs $15 \%$, i.e. they have a greater propensity for readmission than do patients with blunt trauma. This finding may be related to the higher likelihood of penetrating injuries requiring operative intervention.

A corollary finding supporting this theory was that patients undergoing operation on the initial hospital stay were slightly over represented in the UPR patient group (48\% vs 36\%). Conversely, patients not undergoing operation on the index admission were under represented in the UPR group (52\% vs 64\%). These findings mirror those of Battistella's series. Additionally, as in that previous study, there was no major difference identified in the initial mean ISS between the group of all discharged patients and those who required UPR.

A noteworthy number of patients required operation and/or ICU care during the UPR. Over half of those returning who were post-op from an operation on the first admission returned for issues related to an operation on the index admission. Preventable re-admissions related to clinical complications were largely associated with errors in technique or judgment. Brown has proposed a number of factors that may help identify patients at risk for readmission [1]. Many of those factors are present in patients with UPR in this series including patient factors, social factors, operative and postoperative factors and discharge location.

Table 10. Distribution of UPR admitting service.

\begin{tabular}{cc}
\hline Trauma & $54 \%$ \\
Pediatrics & $11 \%$ \\
Orthopedics & $11 \%$ \\
Neurosurgery & $10 \%$ \\
General Medicine & $7 \%$ \\
OB/Gyn & $3 \%$ \\
ENT & $2 \%$ \\
Cardiology & $2 \%$ \\
\hline
\end{tabular}


As they relate specifically to trauma patients, the findings of this study mirror those of Batistella [7]. In that series, $1.4 \%$ of patients required re-admission over $71 \%$ of which occurred within 14 days. Marcin [5] reported somewhat higher rates of re-admission in the non-elderly, 16 65 trauma patient age group (4.7\%) and in elderly trauma patients greater than 65 (9\%). That study did not include re-admissions over 30 days from initial discharge.

Battistella reported that $59 \%$ of patients re-admitted had undergone an operation on the first admission in comparison to $48 \%$ in this series. That study reported a $24 \%$ rate of operation during re-admission which is similar to the $28 \%$ reported in this series. Battistella also found that $29 \%$ of re-admissions were related to wound issues, which is similar to the $26 \%$ noted in this series. The report by Marcin did not detail the reasons for re-admission. There were decidedly less complications related to thromboembolic events and pulmonary issues in this series when compared to the series by Batistella. This difference in thromboembolic complications (19\% vs $1.6 \%)$ resulting in re-admission may be related to a trend towards more attention to, and more aggressive, DVT prophylaxis in the time interval between the two studies.

However, in contradistinction to the prior work, this study revealed an over representation of males in the UPR group ( $86 \%$ vs $70 \%$ ) suggesting there may, in fact, be a male predilection for UPR. This study found no overwhelming difference in mean or median age between the UPR patients and all discharged patients. Additionally, the finding that there were equal proportions of elderly patients in both groups evaluated here point to advanced age not being a risk factor for UPR as previously described. In this study, it also appears LOS on first hospital admission was notably longer in the UPR group compared to the sample of all discharges again differing from Battisella's report that initial LOS was not a risk factor for re-admission. Discrepancies between the two studies may be explained by the large difference in sample sizes or time interval between the two study periods.

Perhaps of note, is the over representation of patients with an ICU stay on the initial hospitalization in the UPR group (57\% vs $40 \%$ ). What appears to be a lengthy average ICU stay for UPR patients requiring intensive care can be explained by one outlier with a stay of 65 days.

Not surprisingly, another finding of this study not reported previously is the over representation of self-pay patients in the UPR group (47\% vs $30 \%$ ). This may be a result of few or no patient resources to obtain appropriate levels of care either as an in-patient at sub-acute facilities or as an outpatient at home. Many self-payers may be the victims of administrative pressure on trauma care providers to discharge these patients as quickly as possible-and perhaps sooner than appropriate-for financial reasons. Conceivably, these attempts at cost saving on the index admission may actually result in "downstream" financial loss due to an inordinate volume of UPR in selfpayers. Therefore, an investment in several further days of stay on the index admission or "pro bono" home health services may garner an overall cost savings. These types of cost-benefit analyses may prove useful.

The vast majority of patients in the UPR group were discharged home. This has been identified as a risk factor for re-admission [1]. Again, this raises the question of greater attention to need, availability and intensity of home services in both payers and non-payers prior to discharge.

Also, while it appears that the performance of any operation is a risk factor for UPR, using the operative index as a gauge, the number of operations per patient is not a major factor influencing UPR. When considering only patients having an operation as a denominator, the operative index for all discharges was 1.6 vs 1.4 in UPR patients. Using the entire number of patients in each group as a denominator, the indices shift but not to a degree that would credibly support the theory that multiple operations on the primary admission is a factor associated with UPR (UPR $=0.7$, all discharges $=0.6$ ). Holloway, in his study of patients discharged from Veterans Administration Medical Centers or private hospitals at VA expense, did show that patients with two or more surgical procedures on the index hospitalization had a greater chance of having an early re-admission [9]. However, his study was not limited to trauma patients and did not discriminate between planned and unplanned re-admissions, but only classified the re-admissions as "early”.

Worthy of comment is that $16 \%$ of UPRs had missed injuries. Of these ten cases, only three represented opportunities for improvement. A previously undiagnosed urethral injury was judged to be iatrogenic and an error in technique. Two were related to systems issues and judgment error. The DVT was asymptomatic and an incidental finding during workup for other symptoms at a rehabilitation facility generating a transfer back and re-admission for treatment. One case was felt to be excusable based on the confounding circumstance of other distracting injuries. In two cases, the suspected injury prompting return was ultimately proven not to exist after further evaluation. Conceivably, these two cases could have been excluded. One case was not initially seen by the trauma service (clavicle Fx) and therefore the missed injury was considered a trauma center, and not specifically a trauma service, issue. Therefore, the categorization of missed injuries may be somewhat overzealous in this analysis. Despite this, the importance of a tertiary survey [10] prior to discharge should not be de-emphasized and would have been potentially advantageous in several of the ten cases.

Unique to this study is the attempt to ascertain prevent- 
ability of re-admission and identify opportunities for improvement which would have obviated the re-admission and its attendant expense and utilization of resources. Unplanned re-admissions have been described as an outcome indicator of quality care in the past in both surgical and non-surgical populations [11]. In this study, overall, $33 \%$ of UPRs were judged preventable or associated with an OFI. The majority were provider related and judged to be a result of an error in technique or an error in judgment. Approximately 20\% were associated with systems issues. The majority of UPRs without evidence for improved care or preventability were disease related, with some being related to systems issues beyond the control of the trauma program.

A study of unplanned re-admissions from a British general surgical population determined that $35 \%$ of re-admissions were avoidable [12]. UPRs were more likely to be judged as avoidable when occurring before 6 days from discharge in comparison to those occurring between 21 and 27 days from discharge (49\% vs $19 \%$ ). Studies have shown varying rates of preventability of re-admission in non-surgical patients ranging from $16.5 \%$ - 33\% [8,10,11].

The determination of preventability or the judgment of opportunities for improvement is subjective and, to some degree, controversial. It has been called into question with particular regard to trauma deaths [13]. Due to the onerous implications of the term "preventable" there has been a trend towards shifting to a taxonomy of complications being promulgated by The Joint Commission which eliminates this term [14]. However, there is evidence to counter fears of discovery and litigation related to discussion of preventable complications in trauma patients. Stewart and colleagues have shown that transparent and open discussion of errors does not increase malpractice risk in trauma patients [15].

Another cogent issue related to this study and others employing judgment panels is the validity and reliability of judgment panel determinations. This applies to both intra-panel and inter-panel reliability. Study population characteristics which influence panels toward, or away from, judging an occurrence preventable have been identified for preventable death studies [16]. It is not clear how these relate to review of complications and UPR.

Taxonomy and fear of litigation aside, identified factors which may have averted the re-admission are amenable to modulation utilizing strategies to educate the provider or the patient. For the provider, these include instruction in proper surgical technique and initiatives to improve judgment. These are generally provided through mortality and morbidity conferences and residency training. However, the extent and quality of such education may be threatened by increasing duty hour restrictions being imposed by the ACGME. Closer supervision of surgical trainees and staff may also be advantageous. For patients, these include education on their conditions and expectations after discharge along with ensuring that discharge instructions including medication use are clearly understood. A robust case management system can also be advantageous in reducing system related UPRs. Finally, the institution of, and compliance with, policies for tertiary survey prior to discharge would effect a decrease in some re-admissions for missed injury [10].

The distribution of UPRs to clinical services other than the trauma service appears to be appropriate. In many instances the reason for admission did not require the global expertise of general trauma surgeons. The conditions precipitating UPR were appropriate to be handled by specialty services, particularly if the patient was operated on by, or transferred to, that specialty service during the initial admission. What did appear to be of some concern after qualitative review of the re-admissions was the fact that several which were relegated to non-trauma services were discovered serendipitously and were felt by the PI workgroup panel to potentially have benefitted from, at minimum, a trauma service consultation.

Several issues seem pertinent to the interpretation of these results. First, is the potential for sample bias. The data are from a single institution. Also, it is not known whether some patients were re-admitted to other trauma centers and/or non-trauma centers for conditions related to their initial hospitalization at the center from which this review emanated. Even at the study institution, complete capture of all trauma patient re-admissions cannot be guaranteed without a sophisticated and burdensome surveillance system. As already mentioned, many re-admissions to services other than the trauma service were serendipitously discovered. Additionally, deaths after discharge from causes related to, or unrelated to, the initial injuries or treatment of them are also not detectable without scrutiny of vital statistics records. Acquisition of such information would require database queries from the state and possibly multiple surrounding states to be all encompassing. Therefore, this study may underestimate or misrepresent the actual rate and nature of re-admissions and their causation.

There are no consistent criteria or protocols for re-admission of trauma or other types of patients after discharge. Therefore, propensity to re-admit is provider, and perhaps trauma center, dependent. Other local sociodemographic factors may also be confounding variables. The same practice related influences may be true for other outcome and clinical measures such as LOS, ICU admission and discharge, etc. This contributes to the potential for wide variation in UPR rates as well as other findings. As noted earlier, changes in practice patterns during the intervening time between studies may also be a factor in interpreting, and drawing conclusions from, these results and comparing them to others. 
Some authors have pointed to importance of the interval of post-discharge surveillance for UPR [17]. These authors cite that UPR rate is susceptible to the choice of time interval and that the identified proportion of re-admissions judged as related to the earlier episode of illness was found to be very sensitive to changes in the observation interval. In general, however, 30 days is considered acceptable and generally thought to be adequate to identify the majority of UPRs after the first hospital encounter. In this series, no UPR occurred after 28 days, and $97 \%$ occurred within 14 days. However the study did not track UPR indefinitely up to the 30-month extent of the review. That fact notwithstanding, this series, and others evaluating the trauma patient population, suggest that most UPRs occurred within 14 days and nearly all within 30 days.

Finally, this study was conducted at a teaching institution. It is conceivable that preventability and OFI rates, along with proportions of technique and judgment errors, may differ at trauma centers that are non-teaching institutions. However, Vachon and colleagues [18] in a similar study performed at a non-academic Level II Trauma Center have reported an almost identical UPR rate of $1.96 \%$. That study also found wound complications to be the leading cause for re-admission with a comparable occurrence of $26 \%$. Also, in that study, $39 \%$ of patients underwent an operation of some sort compared to $28 \%$ in this study suggesting that the incidence of postoperative wound complications may be higher at academic, Level I Centers. However, it is not clear whether the number of traumatic, contaminated, wounds not associated with surgical procedures were similar in both samples. Additionally, the severity, acuity and nature of injuries and operations cannot be adequately compared and controlled for.

In summary, this study of unplanned re-admissions of trauma patients shows results that in many ways are similar to those of previous studies with regard to rate and nature of causes of re-admission as well as demographic and clinical characteristics. It further, however, identifies factors which may be controllable and result in reduction of unplanned re-admission if adequately addressed. This type of information is felt to be valuable for benchmarking purposes and construction of strategies to improve performance. Attention to discharge instructions and education of patients as well as providers, along with a robust case management program and outpatient support services, coupled with a consistent system of tertiary survey prior to discharge, may mitigate a number of preventable unplanned re-admissions. Any efforts to reduce UPRs can effect a corresponding reduction in expense and unnecessary burden on trauma center resources. However, it may be most cost effective to direct these efforts at the subset of patients who have shown to be at high risk for UPR and overrepresented in that group.

\section{REFERENCES}

[1] R. E. Brown, M. Qadan, R. C. G. Martin and H. C. Polk, "The Evolving Importance of Readmission Data to the Practicing Surgeon,” Journal of the American College Surgeons, Vol. 211, No. 4, 2010, pp. 558-560.

[2] D. B. Hoyt, R. Coimbra, B. Potenza, et al., "A TwelveYear Analysis of Disease and Provider Complications on an Organized Level I Trauma Service: As Good As It Gets?” The Journal of Trauma and Acute Care Surgery, Vol. 54, No. 1, 2003, pp. 26-37. doi:10.1097/00005373-200301000-00004

[3] D. N. Ang, F. P. Rivara, A. Nathens, et al., "Complication Rates among Trauma Centers," Journal of the American College of Surgeons, Vol. 209, No. 5, 2009, pp. 595-602.

[4] R. R. Ivatury, K. Guilford, A. K. Malhotra, et al., "Patient Safety in Trauma: Maximal Impact Management Errors at a Level I Trauma Center," The Journal of Trauma and Acute Care Surgery, Vol. 64, No. 2, 2008, pp. 265-272. doi:10.1097/TA.0b013e318163359d

[5] J. P. Marcin and P. S. Romano, "Impact of BetweenHospital Volume and Within-Hospital Volume on Mortality and Readmission Rates for Trauma Patients in California," Critical Care Medicine, Vol. 32, No. 7, 2004, pp. 1477-1483. doi:10.1097/01.CCM.0000127781.08985.03

[6] D. D. Cardenas, J. M. Hoffman, S. Kirschblum and W. McKinley, "Etiology and Incidence of Rehospitalization after Traumatic Spinal Cord Injury: A Multicenter Analysis," Archives of Physical Medicine and Rehabilitation, Vol. 85, No. 11, 2004, pp. 1757-1763. doi:10.1016/j.apmr.2004.03.016

[7] F. D. Battistella, S. Z. Torabian and K. M. Siadatan, "Hospital Readmission after Trauma: An Analysis of Outpatient Complications," The Journal of Trauma and Acute Care Surgery, Vol. 42, No. 6, 1997, pp. 1012-1017. doi:10.1097/00005373-199706000-00004

[8] G. F. Anderson and E. P. Steinberg, "Hospital Readmission in the Medicare Population," The New England Journal of Medicine, Vol. 311, 1984, pp. 1349-1353. doi:10.1056/NEJM198411223112105

[9] J. J. Holloway, S. V. Mendendorp and J. Bromberg, "Risk Factors for Early Readmission among Veterans," Health Services Research, Vol. 25, No. 1, 1990, pp. 213-237.

[10] T. T. Huynh, K. R. Moran, A. H. Blackburn, et al., “Optimal Management Strategy for Incidental Findings in Trauma Patients: An Initiative for Mid-Level Providers,” The Journal of Trauma and Acute Care Surgery, Vol. 65, No. 2, 2008, pp. 331-336. doi:10.1097/TA.0b013e31817e5153

[11] U. Balla, S. Malnick and A. Schattner, "Early Readmissions to the Department of Medicine as a Screening Tool for Monitoring Quality of Care Problems,” Medicine, Vol. 87, No. 5, 2008, pp. 294-300. doi:10.1097/MD.0b013e3181886f93

[12] A. Clarke, “Are Readmissions Avoidable?” British Medical Journal, Vol. 301, 1990, pp. 1136-1138. 
doi:10.1136/bmj.301.6761.1136

[13] D. S. Wilson, J. McElligott and L. P. Fielding, "Identification of Preventable Trauma Deaths: Confounded Inquiries?” The Journal of Trauma and Acute Care Surgery, Vol. 32, No. 1, 1992, p. 45. doi:10.1097/00005373-199201000-00010

[14] A. Chang, P. M. Schyve, R. J. Croteau, et al., "The JCAHO Patient Safety Event Taxonomy: A Standardized Terminology and Classification Schema for Near Misses and Adverse Events," International Journal for Quality in Health Care, Vol. 17, No. 2, 2005, pp. 95-105. doi:10.1093/intqhe/mzi021

[15] R. M. Stewart, M. G. Corneille, J. Johnston, et al., "Transparent and Open Discussion of Errors Does Not Increase Malpractice Risk in Trauma Patients," Annals of
Surgery, Vol. 243, No. 5, 2006, pp. 645-651. doi:10.1097/01.sla.0000217304.65877.27

[16] E. J. MacKenzie, D. M. Steinwachs, L. R. Bone, et al., "Inter-Rater Reliability of Preventable Death Judgements," The Journal of Trauma and Acute Care Surgery, Vol. 33, No. 2, 1992, p. 292. doi:10.1097/00005373-199208000-00021

[17] H. Torhild and S. E. Lilleeng, "Measuring Readmissions: Focus on the Time Factor," International Journal for Quality in Health Care, Vol. 15, No. 2, 2003, pp. 147-154. doi:10.1093/intqhc/mzg019

[18] C. M. Vachon, M. Aaland and T. H. Zhu, "Readmission of Trauma Patients in a Nonacademic Level II Trauma Center," The Journal of Trauma and Acute Care Surgery, Vol. 72, No. 2, 2012, pp. 531-536. 